\title{
LA CONDICION DE LAS MUJERES: EL CASO DE ITALIA DESPUÉS DE LA GUERRA *
}

\author{
Laura Balbo y Marie P. May
}

El artículo estudia las características de la fuerza de trabajo femenina italiana desde los años sesenta en adelante. En comparación con el trabajo femenino en otros países europeos, la mujer italiana trabaja en la producción en menor proporción que en estos países, no se reintegra a partir de los cuarenta años y su trabajo forma parte de las actividades más marginales y peor pagadas.

La razón de esta situación laboral parece encontrarse en las características y funciones de la familia italiana. Esta estructura y funciones se describen para el caso de una categoría social específica, la «capa intermedia», que es mayoritaria en la sociedad italiana.

Finalmente se indican algunos datos relativos a la participación politica y sindical de las mujeres.

* Reproducción de Sociologie et Société, vol. 6-1 (mayo 1974). 


\section{Introducción}

Nos proponemos en este artículo: a) describir las características de las fuerzas de trabajo femeninas, de la organización de la familia y de la presencia de las mujeres en los grupos y las organizaciones que representan los intereses políticos, sindicales, «femeninos»; $b$ ) interpretar el caso italiano introduciendo elementos, incluso parciales y simplemente indicativos, de análisis comparativo con otros países del capitalismo avanzado.

No pudiendo desarrollar un estudio histórico adecuado, analizaremos sobre todo la fase actual: asimismo indicamos esquemáticamente algunos elementos para identificar las principales fases de la evolución del sistema económico y social italiano, a partir de 1945 hasta nuestros días, significativas desde el punto de vista del análisis de la condición femenina:

De 1945 hasta los primeros años 50:

- reconstrucción y primera fase de desarrollo

- paro de masas: alto porcentaje de empleo en agricultura

- bajo nivel de los salarios.

Final de los años 50 - principio de los años 60:

- desarrollo económico sostenido: el «milagro italiano»

- concentración del desarrollo en las zonas urbanas del Norte

- pleno empleo: éxodo masivo de la agricultura: migraciones internas

- crecimiento de los salarios reales.

A partir de la recesión 62.63 hasta el final de los años 60:

- recesión económica y, después, reestructuración industrial

- expulsión de mano de obra femenina, jóvenes y personas mayores

- crecimiento del trabajo a domicilio

- crecimiento de los salarios reales. 
A principio de los años 70 :

- inversión probable de la tendencia: reestructuración económica y crecimiento del empleo

- estabilidad o disminución de los salarios reales.

EI análisis que presentamos aquí toma en consideración las dos últimas fases indicadas, es decir, el curso de los años 60 y el principio de los años 70 , período en el cual los datos son solamente indicativos de las futuras tendencias potenciales. Añadimos incluso que el análisis se interesa en particular por las condiciones propias de las zonas desarrolladas del Norte de Italia; ${ }^{1}$ las características de la Italia del Sur así como la interpretación global de la evolución del país en los términos de dualismo, que le son característicos, no serán estudiadas aquí.

\section{Características de la mano de obra femenina}

El punto de partida para el análisis del trabajo femenino se ha tomado a partir de los datos presentados en los cuadros.

\section{CUAdRo 1}

Porcentajes de actividad según el sexo

\begin{tabular}{lcclcc}
\hline \multicolumn{1}{c}{ País } & Hombres & Mujeres & País & Hombres & Mujeres \\
\hline USA (71) & 53,9 & 30,4 & Alemania (71) & 58,7 & 30,2 \\
Canadá (69 & 53,5 & 24,8 & Noruega (69) & 56,4 & 23,6 \\
Japón (71) & 61,7 & 37,6 & Países Bajos (60) & 56,8 & 16,1 \\
Austria (70) & 53,3 & 30,2 & Suiza (70) & 63,9 & 32,4 \\
Bélgica (71) & 56,1 & 26,1 & Suecia (69) & 60,0 & 37,8 \\
Dinamarca (71) & 59,6 & 37,8 & Inglaterra (70) & 60,4 & 32,2 \\
Finlandia (70) & 55,1 & 37,5 & Italia (71) & 54,1 & 19,2 \\
Francia (72) & 55,0 & 29,7 & & & \\
\hline
\end{tabular}

Fuentes: BIT Annuaire des statistiques du travail 1972, para todos los países indicados excepto Italia (ISTAT Anntario di statistiche del lavoro, 1972, Roma, 1972), Canadá y Suecia (OCDE Bulletin statistique en: ISTAT Annuario di statistiche det lavoro, 1972, Roma, 1972).

1. El análisis presentado en este artículo está basado en la utilización de los 


\section{CuAdRo 2}

Composición porcentual por sexo de las fuerzas de trabajo

\begin{tabular}{lcclcc}
\hline \multicolumn{1}{c}{ Pais } & Hombres & Mujeres & Pais & Hombres & Mujeres \\
\hline & & & & & \\
USA & 62,7 & 37,3 & Alemania & 63,7 & 36,3 \\
Canadá & 67,8 & 32,2 & Países Bajos & 76,2 & 23,8 \\
Japón & 60,6 & 39,4 & Noruega & 69,5 & 30,5 \\
Austria & 61,3 & 38,7 & Suecia & 61,3 & 38,7 \\
Bélgica & 67,4 & 32,6 & Inglaterra & 63,3 & 36,7 \\
Dinamarca & 61,6 & 38,4 & Italia & 73,1 & 26,9 \\
& & & & & \\
\hline
\end{tabular}

Nota: Para todos los países considerados, los datos son relativos al año 1969, excepto para Italia (1971) y para los Países Bajos (1960).

Fuentes: OCDE Bulletin statistique, en: ISTAT Annuario di statistiche del lavoro 1972, Roma, 1972 para todos los paises considerados, excepto para Italia (ISTAT Annuario di statistiche del lavoro 1972, Roma, 1972).

Un primer elemento que caracteriza la situación italiana sobresale de la consideración que, de los 15 países occidentales considerados (cuadro 1) Italia y los Países Bajos tienen el porcentaje de actividad femenina más baja: siete países en efecto tienen porcentajes superiores al $30 \%$, seis, porcentajes comprendidos entre el 20 y el $30 \%$, dos solamente inferiores al $20 \%$, concretamente Italia y los Países Bajos. Se debe añadir además que los porcentajes italianos han descendido a lo largo de los años 60: de $24,9 \%$ en 1960 , desciende a $20,8 \%$ en 1965 y a $19,2 \%$ en $1972 .{ }^{2}$

Un segundo elemento típico del caso italiano está constituido por la diferencia extremadamente acentuada entre los porcentajes de actividad masculina y los porcentajes de actividad femenina. Italia está entre los países en los cuales los porcentajes tanto genéricos (cuadro 1) como por grupos de edad (cuadro 3) son las que están más alejadas de los porcen-

datos estadísticos nacionales y sobre los primeros resultados de una investigación sociológica llevada a cabo en una zona industrializada de Italia del Norte. Ver L. Balbo: «Le condizioni strutturali della vita familiare», y P. May: «Mercato del lavoro feminile: espulsione o occupazione nascosta?», Inchiesta, 9 (1973), pp. 10-37.

2. Los datos, salvo en caso de indicación contraria, han sido extraídos de ISTAT, Annuario di statistiche del lavoro 1972, Roma, 1972, en lo que respecta a Italia; BIT, Annuaire des statistiques du travail 1972, para los otros países. 
«Papers»: Revista de Sociología

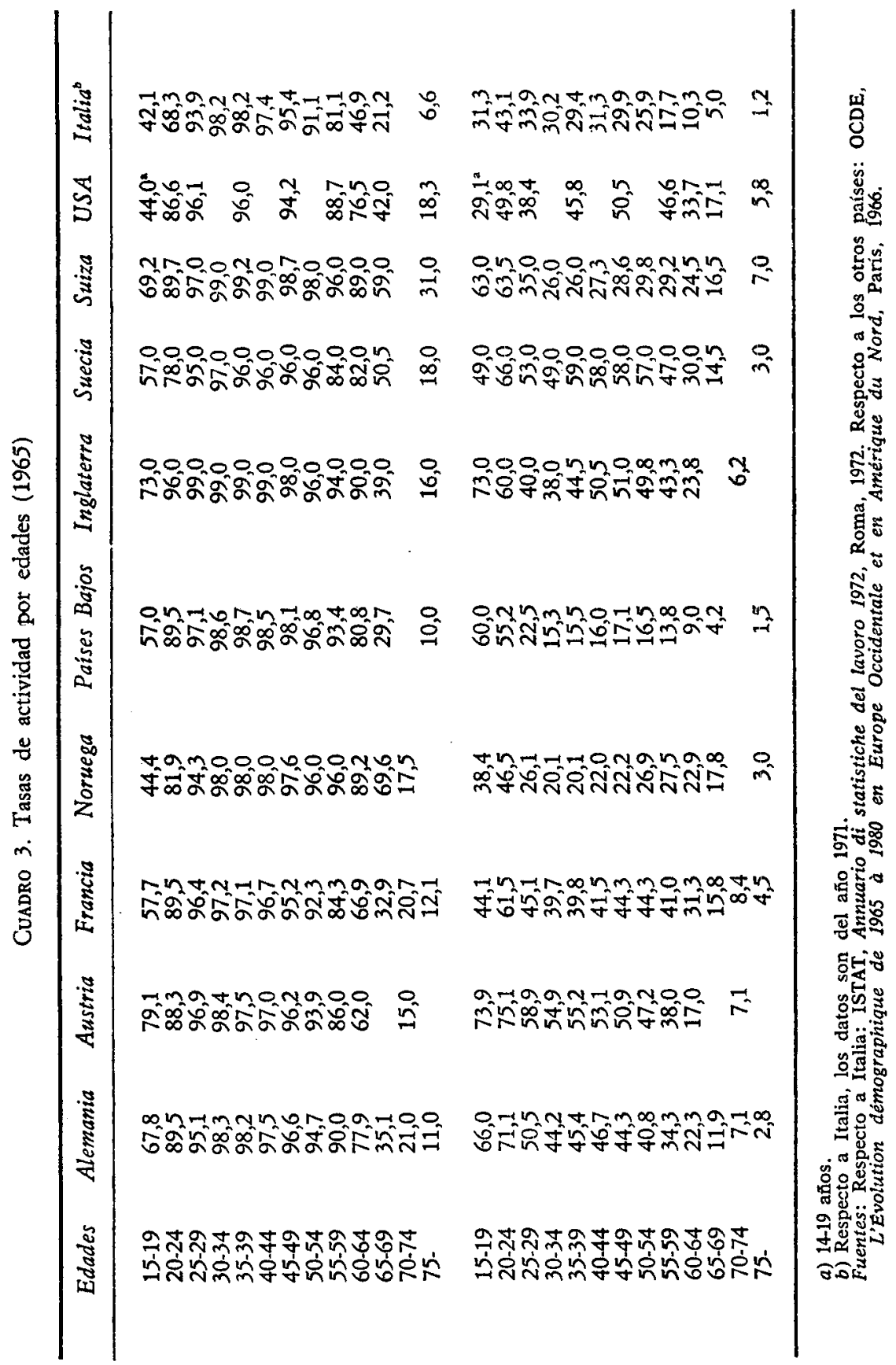


tajes masculinos correspondientes: dicho de otra manera, el mercado del trabajo masculino presenta unos comportamientos que no se diferencian de la situación modal mientras que el mercado del trabajo femenino presenta, comparado con las situaciones modales, una considerable anomalía.

Una medida de tal particularidad se ha dado por la composición por sexos de las fuerzas de trabajo italianas con respecto a las de otros países (cuadro 2): mientras que en 13 de los 15 países industrializados las mujeres constituyen entre el 30 y el $40 \%$ de las fuerzas de trabajo totales (es decir, más o menos una trabajadora por dos trabajadores masculinos), en Italia este porcentaje está en $27,2 \%$ (es decir, un poco más de una trabajadora por tres trabajadores masculinos).

El tercer punto importante a citar aquí es la caracterización no cuantitativa sino cualitativa de la participación femenina en las fuerzas de trabajo. El cuadro 4 explica las curvas relativas a los porcentajes de actividad por grupos de edad en los países de capitalismo avanzado. Mientras que las curvas de los porcentajes de actividad masculina son extremadamente simples y similares para todos los países considerados - un máximo único correspondiente a 35-55 años- las curvas de los porcentajes de actividad femenina son más complejas y más diversificadas entre ellas. El modelo más difundido presenta: dos máximos —en correspondencia con los grupos de edad en los cuales la participación en el mercado del trabajo es más elevada, es decir, el grupo de jóvenes y de los muy jóvenes (15-25 años) y el grupo de mujeres de más de $35-40$ años; un mínimo -en correspondencia con la edad media en la cual la mayor parte de las mujeres se casan y tiene niños. ${ }^{3}$ Con respecto a este modelo, Italia constituye una excepción, incluso en el caso de estar menos marcada de lo que hemos visto en el primero y segundo punto. Después del primer máximo que corresponde a los años más jóvenes, la curva no presenta ninguna subida significativa, sino solamente una bajada continua, más o menos acentuada según los diversos grupos de edad.

Para intentar ceñirnos más a la especifidad del caso italiano con respecto a la situación que aparece como más frecuente en los otros países

3. Las cifras de los porcentajes de actividad según la edad han sido extraídas de una publicación del OCDE relativa al año 1965 (OCDE, L'Evolution démographique de 1965 à 1980 en Europe occidentale et en Amérique du Nord, París, 1966): esta elección está relacionada con el hecho de que mientras que en este estudio son examinados los grupos por edades por períodos de cinco años, otras publicaciones internacionales más recientes examinan por desgracia grupos de edades demasiado extensos para poder permitir un análisis exacto del fenómeno de la salida o entrada sucesiva de mujeres en el mercado del trabajo (los anuarios del BIT, por ejemplo, reúnen en un solo grupo de edades a las mujeres de 30 a 49 años). 
«Papers»: Revista de Sociología

Cundro 4. Porcentajes de actividad por sexo y por edades (Inglaterra e Italia)

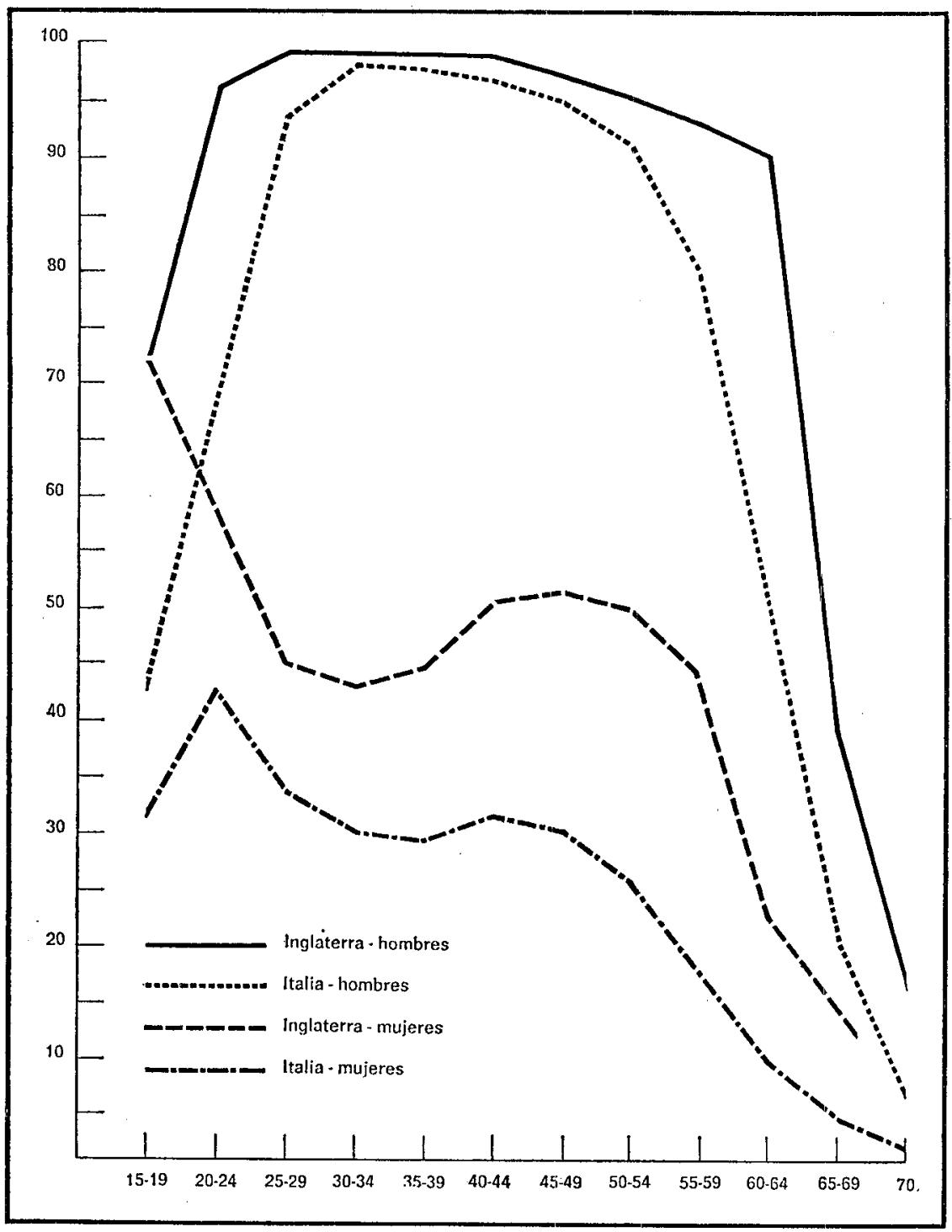


occidentales, vamos a compararla ahora con otra situación concreta, la de Gran Bretaña: país europeo, de dimensión media, de «capitalismo avanzado». El Reino Unido, en lo que concierne al mercado del trabajo femenino representa, sin extremarlas, las tendencias comunes en el área de los países considerados.

Con ayuda de los datos indicados en los cuadros 3 y 4 , el avance profesional de la mujer inglesa y el de la mujer italiana pueden ser descritos de esta manera:

a) Una vez terminados los estudios, las jóvenes inglesas están presentes en el mercado del trabajo ni más ni menos que los jóvenes de su misma edad, si bien el porcentaje de actividad del grupo de los 15-19 años es considerablemente elevado $(73 \%)$ y prácticamente el mismo del de los muchachos. El período siguiente, de 20 a 35 años, en el curso del cual hace su entrada en el mercado del trabajo la parte inactiva restante de la población masculina, corresponde, para las mujeres inglesas, al período durante el cual ellas se casan y tienen niños de edad preescolar (en 1968, el $29 \%$ de las jóvenes se casaron antes de los 19 años y el $85 \%$ antes de los 24 años): ${ }^{4}$ hay en ese momento una salida masiva de mujeres del mercado del trabajo, que disminuye en la mitad la tasa de actividad femenina (30-34 años: $38 \%$ ). A partir de los 35 años, es decir, cuando la mayor parte de las mujeres tienen los niños de edad escolar o de más edad, se constata un retorno considerable al mercado del trabajo, aunque el porcentaje de actividad para las mujeres de 45 a 49 años es superior al $50 \%$, para volver a descender con la llegada de la edad de la jubilación.

b) Si observamos los porcentajes de actividad según la edad en Italia, vemos que, mientras que la curva de los porcentajes de actividad masculina sigue de cerca la curva inglesa, la de los porcentajes de actividad femenina se aleja considerablemente de la curva inglesa correspondiente. Ante todo la presencia de mujeres jóvenes es muy inferior a la de las inglesas de la misma edad: el porcentaje de actividad de 15 a 19 años es, en el caso italiano, muy inferior a los porcentajes ingleses correspondientes $(31,5 \%$ contra $73 \%$ ), y es también inferior a los porcentajes de actividad masculina italiana de la misma edad $(31,5 \%$ contra $42,1 \%)$. El grupo que presenta el porcentaje más elevado corresponde a los $20-24$ años $(43,1 \%)$, después de lo cual la curva desciende más o menos rápidamente, sin nin-

4. Central Statistical Office, Social Trends, núm. 1, 1970. Los datos relativos a otros países confirman este dato: en 1968, el $32 \%$ de las americanas se casaron antes de los 19 años, así como el $25 \%$ de las alemanas y el $21 \%$ de las francesas (ONU, Annuaire démograpbique 1970, Nueva York, 1971). 
guna subida significativa. En dos momentos la disminución es particularmente acentuada, en la edad de la jubilación y en el período del nacimiento y de la presencia de los niños; va incluso más allá del modelo inglés: en 1968, sólo el $11 \%$ de las italianas se casaron antes de los 19 años y el $59 \%$ antes de los 24: además, la mitad de las mujeres que tuvieron su primer niño en este mismo año tenían como mínimo 25 años y una cuarta parte tenían como mínimo 28 años. ${ }^{5}$

En síntesis: en Inglaterra prácticamente todas las mujeres jóvenes entran en el mercado del trabajo, una buena parte de ellas paran de trabajar con el matrimonio y el nacimiento de los hijos, para reemprender más tarde cuando los niños son mayores. El modelo italiano es -al menos en apariencia - más lineal que el inglés: entrada limitada, cuantitativamente, de las jóvenes, a la cual sigue un éxodo continuo de las fuerzas de trabajo femeninas. El problema está pues, en interpretar tanto la tasa reducida de actividad femenina como el fenómeno de la ausencia del retorno de las mujeres casadas.

Diversas hipótesis han sido avanzadas para interpretar las modalidades de la presencia en el mercado del trabajo no sólo de las mujeres sino también de todas aquellas partes de la población que aparecen como «débiles» con respecto al núcleo central de la oferta de trabajo, constituido fundamentalmente por los hombres del grupo de la edad de 30-50 años, es decir, además de las «mujeres», los «viejos», los «jóvenes», los «subnormales».

De todo ello ha derivado un debate en el cual han participado (y participan) economistas y sociólogos. ${ }^{6}$ Hemos estudiado solamente aquí, extremadamente esquematizadas $-\mathrm{y}$ en consecuencia también simplificadaslas hipótesis principales: a) una gran parte de mujeres se ha retirado voluntariamente del mercado del trabajo como consecuencia de una mejora en sus condiciones de vida, de la elevación de los niveles de instrucción y de la difusión de los modelos de vida, de la clase media; $b$ ) una gran parte de mujeres se ha retirado, si se quiere, voluntariamente, no para escoger el rol de la mujer en el hogar en sus aspectos positivos, sino más bien para escapar al destino de la mujer que trabaja, en una fase de reestructuración de la economía italiana que ha comportado la intensificación de los ritmos de trabajo, la competencia por parte de los jóvenes inmigrados

5. ISTAT, Anuario di statisticbe demograficbe 1968, Roma, 1971.

6. Los ensayos más significativos aparecidos recientemente en Italia relativos al debate sobre el mercado de trabajo han sido recogidos: ver P. Leon y M. Marocchi, Sviluppo economico italiano e forza-lavoro (Padua, Marsella, 1973). 
y de los largos horarios de trabajo; c) una gran parte de mujeres ha sido expulsada del mercado del trabajo a raíz de la crisis que se originó a principios de los años 60 . No se trata en ningún modo de una acción voluntaria y aceptada, sino de situaciones de «inactividad solapada», que los criterios seguidos por los datos estadísticos italianos no permiten descubrir.

Sin entrar en detalles de estas interpretaciones, es suficiente señalar como hecho central la disminución de la participación femenina en las fuerzas de trabajo: el punto central en el cual se insiste más es que una gran parte de mujeres - y particularmente de mujeres de más de treinta años- no están ya en el mercado del trabajo.

Estas conclusiones parecen quizás contrastar con las indicaciones sugeridas sobre la condición efectiva del mercado del trabajo en algunas ocasiones y a través de fuentes diferentes (resultados de encuestas hechas por el Instituto Central de Estadística sobre la población «inactiva»: investigaciones sobre el trabajo a domicilio en algunas regiones italianas; comparación incluso con las características del empleo femenino en los otros países capitalistas). Sobre la base de estos elementos, ciertamente insuficientes para sacar conclusiones definitivas, es sin embargo posible dudar de que la parte de las mujeres inactivas y que se quedan en casa sea la indicada por las cifras oficiales y de llegar a la hipótesis de un fenómeno de verdadero "empleo escondido", localizable sobre todo en este grupo de edades que en otros países manifiestan una entrada importante de las fuerzas de trabajo. Salidas o expulsadas del sector central del mercado del trabajo (caracterizado por el «pleno rendimiento», por la estabilidad del empleo, por las garantías del seguro y de la prevención; pero también por la rigidez de los horarios, por la insuficiencia de las ausencias legítimas y de las vacaciones - y en consecuencia por la ausencia de flexibilidad frente a las exigencias serias de los diferentes grupos del medio de trabajo) las mujeres entran en el mercado del trabajo en una situación marginal. ${ }^{7}$

7. Lo que se entiende exactamente por trabajo marginal en la literatura sobre el mercado del trabajo capitalista está aún por precisar conceptualmente y por describir en todas sus articulaciones concretas. Una primera contribución ha sido aportada, refiriéndose a la situación en los Estados Unidos, por Dean Morse, el cual define el trabajo marginal o periférico como una categoría de residuos, en oposición al trabajo estable y de «dedicación completa». Según Morse, «la fuerza-trabajo periférica constituye una categoría extremadamente heterogénea», de la cual el elemento crucial de la definición es que «este grupo está unificado solamente por el hecho de que, por una razón u otra, sus miembros no escogen o están obligados a no escoger el tipo de trabajo de dedicación completa». D. Morse, The Peripberal Worker (Nueva York y Londres: Columbia University Press, 1969), p. 46. 
El proceso del trabajo típico de la mujer italiana - hablamos aquí solamente del trabajo no agrícola y de las zonas urbanas industrialesse presentaría desde entonces así: entrada en el mercado del trabajo de los jóvenes y de los muy jóvenes; salida del mercado del trabajo de la mayor parte de las mujeres presentes en el momento del matrimonio o del nacimiento de los hijos; para una parte de las mujeres la salida es definitiva, para la otra, es provisional y la rentrée se produce para la mayoría en el área del mercado marginal. En los demás países también una gran parte de mujeres, sobre todo las que entran en el mercado del trabajo después de haber criado a sus hijos pequeños, trabajan según las características que son propias del trabajo marginal, y en particular se prestan a actividades a «tiempo parcial». Por otra parte, ningún país occidental ha resuelto ni ha intentado resolver el problema del trabajo femenino de una manera radical. En las situaciones más avanzadas se han introducido facilidades de horarios, horas de servicio, vacaciones, ausencias motivadas, cualificaciones, mejoramiento de algunos servicios sociales, etc. Pero de todas maneras el mercado del trabajo femenino es siempre distinto del masculino y entra en su mayor parte dentro del área del mercado marginal.

Lo que caracteriza la situación italiana es que la parte más importante del trabajo marginal femenino está constituida por el «trabajo negro», sobre todo bajo la forma de trabajo a domicilio. Considerado hasta hace unos años como una relación de producción que quedó del paso del capitalismo italiano de sus formas arcaicas a sus formas modernas, y destinado por lo tanto a desaparecer, el trabajo a domicilio aparece por el contrario, en el curso de los años 68-72, en rápido crecimiento. Una estimación relativa únicamente a las industrias manufactureras indica para Italia una cifra entre 1.015 .000 y 1.505 .000 y para la Lombardía entre 230.000 y 275.000 . Las mujeres constituirían entre el $77 \%$ y el $90 \%$ del total, según las zonas y las ramas productivas. Siguiendo con la Lombardía los salarios - teniendo en cuenta la amortización, el cuidado de las máquinas a cargo del trabajador, la ausencia de indemnizaciones complementarias: vacaciones pagadas, etcétera-, están en una media a nivel del $60 \%$ de los salarios pagados como mínimo contractual a los trabajadores ocupados en las fábricas: el horario medio es de 8 horas y media por día y de 48 horas a la semana (pero en algunos casos, incluso 13-15 horas y 55 horas): los ritmos de trabajo son más altos que los tolerados en las fábricas, donde existe un control sindical: finalmente, no hay ningún tipo de seguridad social. ${ }^{8}$ Total que se trata de un trabajo marginal que tiene unos costes particularmente elevados.

8. Los datos relativos al trabajo a domicilio están extraídos de una investigación 


\section{Estructura y funcionamiento de la familia}

Vamos a introducir aquí algunos datos relativos a la variante fundamental para el análisis de la condición femenina: la estructura y el funcionamiento de la familia. En este contexto, nos limitaremos al análisis de la familia en sus consecuencias respecto a la condición de la mujer: en la familia en sí misma, en el trabajo, en la experiencia de la participación sindical, política, o en los nuevos movimientos feministas. En el sistema italiano los elementos fundamentales a considerar son los siguientes:

1. Existe un conjunto de tareas relativamente rígidas y bien definidas (rígidas en el sentido de que son parecidas, y obligatorias en todas las familias; bien definidas en el sentido que existe un consensus fundamental sobre la definición de las tareas) que son atribuidas a la familia. Esquemáticamente: $a$ ) tareas relativas a sobrevivencia y al bienestar de sus miembros, principalmente de los niños que dependen de ella; $b$ ) tareas relacionadas a la compra y al mantenimiento de los bienes de propiedad y de uso de la familia: la esfera del consumo y las actividades que le son relativas; c) tareas, que nosotros llamaremos burocráticas, que derivan del hecho que la familia es una unidad jurídica y administrativa: pagar los impuestos, inscribir a los niños en la escuela, encargarse de las vacunas y los controles médicos, etc., son en efecto tareas burocráticas.

Algunas reglas, jurídicas en parte pero sobre todo culturales, determinan este conjunto de tareas: lo esencial es que ninguna familia, que funcione como tal, no pueda sustraerse definitivamente y sistemáticamente de la obligación de cumplirlas.

2. Todos los elementos concretamente utilizables para desarrollar las diferentes funciones que le son atribuidas son los recursos de que disponen las familias. Y por lo tanto, la presencia y las capacidades particulares del padre y de la madre, y de otros familiares eventualmente disponibles; los bienes y los servicios que se compran, a partir del alquiler hasta los aparatos eléctricos de la casa, de las comidas cocinadas y las horas de guardería, los servicios prestados por el sistema público, tales como los servicios médicos y de asistencia o la escuela. Hay que hacer una distinción fundamental entre los servicios privados y los servicios públicos: es la que sobresale en la caracterización siguiente de los recursos . que permiten la gestión familiar:

a) Recursos propios de la unidad familiar: en Italia como casi en todas partes, la familia nuclear es el modelo y, por consecuencia, los miem-

hecha en 1972 en la Lombardía, cuyos resultados han sido publicados en L. Frey, I problemi del lavoro a domicilio in Lombardia (Milán, 10 julio 1972). 
bros adultos presentes son sólo el padre y la madre: el padre, proveedor, con sus ganancias, de las necesidades de la familia; la madre, principal responsable de la gestión de los recursos para el cumplimiento de las tareas.

b) Recursos externos a la familia, disponibles en el mercado privado. Muchas de las funciones que en el pasado eran asumidas por la familia (producción de bienes, funciones de servicio, funciones recreativas, la so cialización incluso de los niños) han pasado en la sociedad actual al exterior. El mercado privado abastece prácticamente de todos los bienes y los servicios que pueden ser integrativos o sustitutivos de los recursos internos de la familia: vestidos de confección, alimentos congelados, escuelas privadas, casas de vacaciones, horas de las asistentas, o de los servicios de guardería.

c) Recursos fuera de la familia, distribuidos por una empresa pública a bajo precio o gratuitamente. Vista la distancia que existe entre las necesidades y la posibilidad de acceso a los servicios del mercado privado, ciertos bienes y servicios considerados como esenciales han sido abastecidos y asumidos progresivamente por el Estado o por las empresas públicas. Un elemento característico de la situación italiana es que los servicios públicos de la asistencia social no provienen del derecho del ciudadano como tal, quien se encuentra en un estado de necesidad, sino que provienen de la existencia misma de una relación específica de asistencia. Si, en el curso de los últimos años, la parte de la población que ha quedado cubierta por estas relaciones ha crecido mucho, queda todavía una parte de la población que no ha sido cubierta por la asistencia pública en una parte de sus necesidades fundamentales. ${ }^{9}$

La estratificación: tres condiciones de vida familiar

La gestión familiar pone en relación el conjunto de tareas con la disponibilidad de los recursos públicos y privados. El acceso a los recursos está determinado, como hemos visto, por la disponibilidad de los ingresos y por la condición que ocupa la familia en relación con el sistema de asistencia pública: por la acción de estos dos criterios las familias han sido diferenciadas y estratificadas.

9. Un estudio reciente estima que el $85 \%$ de la población italiana está cubierta por la seguridad social. Ver A. Ceci y R. Proietti, «Il carico familiare nelle programmazione economica regionale», Rivista italiana di economia demografica e statistica, 1968, núm. 1-4. 
a) Las familias con altos ingresos pueden «comprar» a un adulto que realizará una parte de las tareas domésticas, modificando así el modelo a partir del cual los padres sólo están disponibles para la gestión familiar: estas familias pueden acceder al mercado privado para una serie de bienes y servicios, habitar en los mejores barrios, tener una casa para las vacaciones, guardería para los niños, asistencia médica en clínicas privadas -eventualmente en Suiza o en los Estados Unidos- instrucción todavía en escuelas privadas, o en el extranjero. Las familias que tienen libre acceso a estos servicios privilegiados y que tienen pues a su disposición unos ingresos adecuados son una pequeña minoría: familias de la burguesía bienestante, ciertas categorías de altos cuadros industriales, funcionarios o miembros de profesiones liberales. Ya que nos interesa dar aquí sólo una indicación cuantitativa lo más amplia posible, estimamos que no más de un $5 \%$ de familias entran en esta categoría.

b) En el extremo opuesto se encuentran las familias cuyos ingresos son apenas suficientes para las necesidades elementales de sobrevivencia, incluso en los casos que las modalidades en vigor en la utilización del sistema de asistencia pública no les aseguren su protección: muchos trabajadores de la agricultura, de la construcción, de los servicios domésticos, del trabajo a domicilio - es decir, justo aquellas categorías que tienen ingresos irregulares y bajos- están por lo menos en parte excluidos o de cualquier manera discriminados en el uso de los servicios públicos. En estas familias, las necesidades no menos importantes que las de tener un techo, de comer, de vestirse, estando éstas incluso más o menos satisfechas, no están cubiertas: son las necesidades relativas a la asistencia médica, a la asistencia especializada (subnormales, ancianos, inválidos), a los servicios recreativos y deportivos, a la instrucción..$^{10}$

c) La gran mayoría de familias se alinea en una «capa intermedia», caracterizada: de una parte por los ingresos suficientes no sólo para las necesidades esenciales, sino también asegurando el acceso al mercado privado para algunos servicios juzgados indispensables, y de la otra por el acceso generalizado a los servicios públicos. Están comprendidas en esta capa las familias cuya condición profesional del jefe de familia, y por consecuencia de los ingresos, es muy diferente: la mayoría de los empleados

10. Una clasificación bastante parecida a la que se adopta aquí para distinguir la capa intermediaria, es la del Bureau of Labor de los Estados Unidos, que distingue un nivel «inadecuado» de un nivel «modesto pero adecuado» de gestión de las necesidades familiares. Para un análisis completo de los problemas de definición y de dimensión de las necesidades familiares, ver Margaret Wynn, Family Policy (Nueva York: Pen guin Books, 1972). 
de diversos niveles, categorías obreras de nivel superior, pequeños y medios comerciantes y empresarios, ciertas categorías de profesiones liberales. La característica común que las distingue de la capa superior es la imposibilidad de recurrir permanentemente y para todas las necesidades familiares al mercado privado: viceversa, al contrario, las familias de la capa inferior, tienen asegurado el acceso a los servicios públicos y pueden, para una parte al menos de sus necesidades, acceder al mercado privado. Las combinaciones concretas posibles entre los recursns públicos, los recursos privados fuera de la familia y los recursos de la familia son evidentemente incalculables: la condición común es la posibilidad de intentar alcanzar un nivel de gestión familiar adecuado.

De las tres condiciones que esquemáticamente hemos esbozado, desarrollaremos el análisis limitándonos a las familias de la «capa intermedia». No se trata sólo en efecto de la capa que comprende la gran mayoría de las familias, sino que desde el punto de vista de la condición de la mujer es allí donde se manifiestan las variantes más significativas en la explicación de lo que se ha puesto en evidencia anteriormente: la ausencia, o mejor la presencia en condiciones especiales, de la mujer en el mercado del trabajo.

\section{La condición de la mujer en la vida familiar}

Para describir la condición de la mujer en la familia de la «capa intermedia» es aún necesario analizar un elemento fundamental de la estructura de la familia: el rol del marido-jefe de familia como rol rígido de trabajador.

Los elementos que caracterizan a los roles masculinos y femeninos en las familias de la sociedad industrial han sido analizados por Talcott Parsons en temas que son aplicables en sus grandes líneas al tipo de familia que consideramos aquí. En la situación italiana específica que nos interesa, es decir, aquella de las áreas urbanas industriales durante los años 60 , el rol de trabajo del jefe de familia tiene las características siguientes:

- Horario de trabajo de ocho horas (o más en los frecuentes períodos en los cuales tiene que hacer horas suplementarias); trayecto casalugar del trabajo de una, dos o incluso tres o cuatro horas cada día. El jefe de familia está pues ausente de la casa en un mínimo de nueve horas y un máximo de catorce horas, o incluso más.

- Período limitado de vacaciones: 10-15 días como media; para una minoría hasta un mes: de todas maneras este período corresponde a una 
pequeña parte de la totalidad de las vacaciones escolares de verano de los niños (más de tres meses).

- Condiciones de trabajo (comprendidas aquellas que se refieren al trayecto casa-lugar de trabajo) muy pesadas tanto en el sentido físico como en el sentido psíquico. La consecuencia de todo esto es que el hombre se identifica principalmente con el mundo de su trabajo, en un sentido a veces pasivo o conflictivo, pero en todo caso teniendo muy poca participación en la vida familiar. Todos los tipos que se encuentran concretamente -el obrero que acepta las horas suplementarias para aumentar su salario, aquel que después de la jornada de trabajo se deja caer delante de la televisión, el militante comprometido en la actividad política o sindical- son igualmente marginados o excluidos de los roles de la gestión familiar. Simétricamente, toda la gestión de las tareas familiares cae sobre la madre, y su rol es a su vez rígido.

El rol de la mujer en el hogar

Se sabe muy bien cuál es el conjunto de tareas que está ligado al cuidado de los niños. Para cumplir estas tareas, la mujer tiene hoy, en la sociedad italiana, unas ayudas muy limitadas. Para la mayoría una ayuda pagada es absolutamente inaccesible, siendo el número de guarderías en absoluto insuficiente, las escuelas primarias tienen un horario de cuatro horas al día solamente (mañana $o$ tarde); muy pocas son las organizaciones, sean privadas o públicas, deportivas o culturales, para niños y adolescentes. La madre italiana tiene, pues, tareas de vigilancia, de organización y de asistencia de los niños durante un período que no está limitado a la edad preescolar y de la escuela primaria (como podría ser el caso en condiciones diferentes del sistema escolar y de los tiempos de ocio en la ciudad).

Una segunda serie de actividades está ligada a los trabajos de la casa. En las familias de la «capa intermedia», el uso de los aparatos eléctricos domésticos, de los alimentos congelados, etcétera, se ha difundido en los últimos años, pero con relación a otros países este uso está todavía relativamente limitado por condicionamientos económicos y culturales. ${ }^{11}$

Al mismo tiempo se constata que las tareas burocráticas de la familia aumentan: la mujer de la familia obrera, la mujer del empleado, del comerciante, del profesional, tiene numerosas tareas relativamente nuevas (o quizás es la manera de realizarlas que es nueva, convirtiéndose así en «burocrática»): tiene que pagar las facturas del gas y de la electricidad,

11. M. Wynn, op. cit. (1972), p. 26. 
el alquiler, los seguros sociales de la asistenta y las multas del coche; debe perseguir las rebajas en los diferentes períodos del año, pensar en los vencimientos de sus compras a plazos, debe ocuparse de-los vencimientos fijados de eventuales empréstitos de los bancos, de los controles médicos de los niños y de sus relaciones con la escuela.

Son los vencimientos rigurosos, las modalidades formales de todas sus tareas las que determinan su particular naturaleza. Así pues lo que importa destacar es que en estas condiciones la presencia de una persona a pleno rendimiento en la familia es indispensable; y esta persona es la mujer.

En este sentido, la «profesionalización» del rol de la mujer en el hogar parece ser más elevada que las condiciones de trabajo del jefe de la familia, que son en sí mismas más rígidas teniendo además recursos disponibles para la gestión familiar menos adecuados. En este cuadro, como veremos, el rol especializado de «ama de casa» aparece con unas características excepcionalmente rígidas en Italia en relación con otros países. En efecto, mientras que la rigidez del rol ocupacional del jefe de familia es un rasgo común en las sociedades del capitalismo avanzado de Occidente y en las sociedades industriales de modelo soviético, la disponibilidad de los recursos varía. En los países de la Europa del Este y en la Unión Soviética (donde las tasas de participación femenina en el mercado del trabajo son muy elevadas), los servicios públicos están considerablemente desarrollados y su acceso está generalizado. En las sociedades capitalistas como los Estados Unidos o Inglaterra, para tomar dos ejemplos, el rol de la mujer en las familias de clase media está ciertamente muy especializado, pero en él se encuentran dos diferencias fundamentales con respecto a la situación italiana: primero, un número creciente de mujeres que vuelven al trabajo después de una interrupción (durante la cual están en el hogar con plena dedicación); segundo, las condiciones generales de vida y los servicios familiares para las familias de la clase media y de la clase obrera superior son indiscutiblemente mejores que las del caso italiano: sin embargo, la especialización y la rigidez del rol de la mujer comportan unos costes inferiores.

Nos referimos aquí a los numerosos análisis de la condición de la mujer en la vida suburbana (es decir, mujeres de la «capa intermedia»: clase media y niveles superiores de la clase obrera): condición evidentemente rígida y especializada, que tiene unos costes elevados para la propia mujer y para los otros miembros de la familia -en especial las modalidades difíciles de la socialización de los niños-, pero condición que aparece sin ninguna duda como menos grave que la italiana, donde los defectos de 
los servicios públicos y las lamentables condiciones de la vida urbana pesan sobre las familias de cualquier condición social.

Resumiendo, pues, lo que es el resultado principal de los elementos considerados hasta aquí: en condiciones similares el becho de que las mujeres madres de familia estén en su mayoria ausentes del mercado de trabajo (o que, si tienen un trabajo, están en empleos a destajo, ocasionales, a domicilio, breves, marginales) es un becho estructural, es decir, no ligado a opciones individuales, becho permanente y generalizado.

\section{Participación política y sindical de las mujeres}

Los datos esenciales relativos a la participación política y sindical de las mujeres son los siguientes:

En una primera fase, desde el final de la guerra hasta los años 50, la participación de las mujeres, particularmente de las mujeres de la clase obrera, en las organizaciones de masa ligadas a los partidos de izquierda ha sido considerable. La organización femenina más importante era la Unión de las Mujeres Italianas (UDI), constituida en 1944 como estructura paralela al Partido Comunista: la UDI organizaba, hacia 1950 a un millón de mujeres aproximadamente, y las movilizaba tanto sobre los temas específicos de la condición femenina (protección de la mujer trabajadora, servicios de asistencia) como sobre los temas políticos en general (campañas para la paz). Además, durante este período, la participación de las mujeres en las organizaciones y en las luchas sindicales fue, para ciertas categorías y en ciertas zonas del país, muy importante y de gran combatividad. ${ }^{12}$

Entre mediados de los años 50 y el final de los 60, las organizaciones existentes y las luchas sindicales conocen una fase de debilitamiento pronunciado (en lo que concierne al comportamiento de participación, se aplica siempre a todas las categorías y a todos los niveles). La UDI, para dar una indicación específica, encuentra resistencias cada vez más fuertes en sus campañas para las inscripciones y a principios de los años $60 \mathrm{el}$ número de sus afiliadas alcanza cerca de 200.000 .

A partir de los primeros años 70 se percibe un aumento de la pre-

12. El número de mujeres inscritas en el PCI durante este período oscila alrededor del medio millón; los datos relativos a la participación sindical femenina no los tenemos. Las fuentes principales en lo que concierne a la participación política de los años 1945-1946 están constituidas por una serie de volúmenes, Ricerche sulla partecipazione politica in Italia (Bolonia: Il Mulino, 1967-1969). 
sencia de las mujeres en la afirmación y en la lucha tanto para los intereses específicos de su condición, como para los objetivos más generales ligados a la sociedad en que viven.

Se trata de tendencias muy recientes y de fenómenos cuyo conocimiento es todavía insuficiente, o de cualquier forma limitado a círculos de opinión aún muy restringidos. Parece importante sin embargo señalar que desde los movimientos de los años 1966-1969 y de las luchas recientes de los estudiantes y de los obreros, es necesario, para analizar los fenómenos de participación, introducir definiciones diferentes y nuevos indicadores.

En particular las formas de expresión del interés de las mujeres, es decir, en Italia, las posiciones feministas, importadas del extranjero pero elaboradas de manera relativamente autónoma, y los movimientos de base en los cuales las mujeres participan activamente, para el alquiler, para los servicios públicos, para las escuelas, deben ser estudiados como un fenómeno profundamente diferente de la participación de los años 40 y 50 . Diferente a causa de sus numerosos componentes de «comportamiento colectivo", y diferente a causa del tipo de población interesada, en su mayoría mujeres de clase media y de niveles superiores de la clase obrera (es decir, formando parte de la «capa intermedia»).

Sobre la base de estas consideraciones el análisis de los datos del pasado parece, pues, poco importante, mientras que toda tentativa de comprensión de las tendencias futuras relativas, no sólo referentes a la condición de la mujer, sino también a la situación general de la sociedad italiana, no puede hacerse sin estos elementos nuevos, incluso los de más reciente aparición.

\section{Conclusión}

Los datos que hemos analizado muestran una coherencia significativa entre los diferentes aspectos del sistema social en general. En un capitalismo incapaz de hacer reformas, como ha sido el capitalismo italiano de los años 50 y 60 , un considerable conjunto de tareas ha sido transferido a la familia; en efecto, como consecuencia del proceso de desarrollo, los standards de satisfacción de las necesidades llegan a ser más elevados en la capa creciente de individuos y de familias; pero esta situación difiere de aquella de otros países donde el proceso de desarrollo se ha acompañado de una política de intervención pública (que nosotros sintetizamos en el modelo del Welfare State). El sistema público italiano no sólo no se ha adaptado a las nuevas necesidades, sino que ha llegado a ser, en un sentido relativo, 
más insuficiente e inadecuado. Más específicamente, siguiendo nuestro tema, la condición de la mujer se ha caracterizado de esta manera: a) su presencia con plena dedicación en la familia como organizadora de los recursos se ha hecho necesaria, y por consecuencia su presencia en el mercado del trabajo ha sido subordinada a esta necesidad fundamental; $b$ ) como consecuencia tanto de la disminución de la presencia de las mujeres en el trabajo y en las organizaciones sindicales, como de la creciente privatización de su esfera de acción, las mujeres han sido y son excluidas de cualquier forma de participación en la vida pública.

Lo que señalamos como conclusión y al mismo tiempo como indicación para posibles estudios posteriores, es que el equilibrio alcanzado en los años 60 entre la estructura familiar, las modalidades del trabajo femenino y los valores privados, no podrá ser el mismo en el futuro. Han surgido llamadas a un cambio radical, en parte como efecto contradictorio de las condiciones de los años 60 , en parte como resultado de factores externos, propios del funcionamiento general del sistema económico y político italiano. Más específicamente, se puede señalar un cierto número de elementos de cambio.

Un elemento de fundamental importancia es la difusión en Italia (con retraso con respecto a otros países capitalistas de Occidente, pero del cual se puede prever un aumento rápido en un futuro inmediato) de las técnicas de control de nacimientos, y por consecuencia la posibilidad por parte de las mujeres de planificar su vida (número de hijos $\mathrm{y}$, sobre todo, el momento de la procreación). Este elemento tendrá una incidencia directa sobre las modalidades de la oferta en el mercado del trabajo de la mano de obra femenina y además, evidentemente, tendrá consecuencias muy importantes para las costumbres y los valores.

Diversos elementos como la elevación de los niveles de escolaridad de la población femenina, la actitud en favor del trabajo estable en las mujeres (habiendo conocido en su gran mayoría, cuando eran más jóvenes, las experiencias del trabajo estable), las reivindicaciones de los sindicatos y de organizaciones como la UDI que se oponen al trabajo a domicilio o a destajo. Estos elementos podrán influenciar las condiciones de «rigidez»: las mujeres no estarán nunca más dispuestas, como en el pasado, a aceptar las formas de trabajo «marginado».

El desarrollo previsible de algunas industrias que ocupan a la mano de obra femenina, como son la electrónica, la química «perfeccionada», la distribución, tendrán una gran influencia en estos cambios.

A nivel de las condiciones de vida familiar, el elemento específico que, a partir de 1970 , se ha acentuado más, es el crecimiento del coste de la vida. Por consecuencia, la explotación de los recursos privados y la 
rigidez del rol de la mujer en el bogar ban llegado a ser cada vez más insostenibles.

Dos elementos, finalmente, hay que recordar a propósito de la presencia de la mujer como sujeto de interés y de reivindicación política. El primero es que se perciben ahora algunas modalidades nuevas de participación, sea en organizaciones nuevas como son los grupos feministas, sea en organizaciones tradicionales, como la UDI. Muy importante será el análisis de las relaciones que se podrán establecer entre el movimiento feminista y fuerzas políticas como los partidos, los sindicatos, la UDI. El segundo es que en Italia las condiciones de vida familiar son objetivamente tan penosas para la gran mayoría de las mujeres (que nosotros hemos definido, en las áreas urbanas del Norte del país, como las mujeres de la «capa intermedia») que determinan unas tensiones y unas formas de participación nuevas: luchas urbanas, luchas para conseguir mejorar los servicios, luchas para conseguir escuelas.

Todo esto no son más que indicaciones. Nos limitamos a decir, en este momento, que este conjunto de elementos nuevos parece indicar que las contradicciones propias de la condición femenina no podrán ser nunca más estudiadas según el modelo de los años 60 . Se puede, pues, pensar en dos alternativas: o un nuevo sistema de equilibrio se implantará, el cual llevará la situación italiana, gracias a una política de reformas en los sectores fundamentales, a acercarse a la de los países de capitalismo avanzado, o se determinarán con relación a las condiciones particularmente atrasadas que existen en Italia, unos comportamientos conflictivos e innovadores. Estos comportamientos serán el resultado de una toma de conciencia específica de las mujeres, pero también de una toma de conciencia más general.

En este sentido el estudio del caso italiano, tal como lo hemos propuesto aquí, podría ser significativo no de una situación «atrasada», sino más bien de un itinerario particular de «desarrollo» capitalista, en el curso del cual las soluciones originales a las contradicciones propias de la condición de la mujer podrían desaparecer. 